\title{
Design and Evaluation of a Large Interactive Display to Support Social Interaction at Work
}

\author{
Sanjin Pajo, Senka Zubić, Agnieszka Matysiak Szóstek, and Janienke Sturm \\ Eindhoven University of Technology, Den Dolech 2, 5612 EZ, Eindhoven, The Netherlands \\ \{S.P.Pajo,Z.S.Zubic, J.Sturm, A.Matysiak\}@tue.nl
}

\begin{abstract}
Social interaction at work has been shown to enhance creativity and productivity. This paper describes the design and evaluation of a large, interactive display that aims to stimulate social interaction amongst co-workers by providing food for talk in the form of activities, jokes, and music. User and system requirements were collected using Cultural Probes and Focus groups. The evaluation of our prototype system provides valuable insights that may guide the design of future systems supporting social interaction at work.
\end{abstract}

Keywords: Design, Large Touch-Screen Display, Social Interaction, BSCW.

\section{Introduction}

In recent years, much research has shown that social interaction can lead to a sense of cohesiveness and cooperation amongst employees. It can, also improve self-esteem, mental health and overall personal satisfaction by reducing anxiety and uncertainty [4]. Social interaction in the workplace can make the job more enjoyable and therefore, enhance creativity and productivity [2]. Moreover, social interaction has been shown to reduce stress and help build up relationships, which can lead to better access to resources and materials [1]. Our goal was to design a large interactive display that is specifically aimed at facilitating and stimulating social interaction among coworkers. This system will be referred to as the LID (Large Interactive Display) system. We elaborate and report on the initial set of requirements that was collected by using methods such as a Cultural Probes and Focus Groups [3] [5]. We also discuss a prototype system and describe the results of a first user test of this system.

\section{Eliciting Requirements}

The first step of the design process was to define the user and system requirements for the design. We decided to use cultural probes [3] as a requirements elicitation method, because they provide not only events but also people's feelings and impressions regarding interactions and preferences as to what parts of their social interactions could be supported by the system. For the cultural probes study we invited 13 participants from two companies: 10 from a small consultancy company and three from a research institute (8 male and 5 female). The study was carried out over a period of five working days. The materials that could be used by the participants (i.e. diary, posters for 
illustrations and cameras) were designed to be engaging, to stimulate thought, and to capture emotions and experiences in a playful manner.

The information gathered from the probes was categorized and put into four main clusters: social awareness, ice breakers, cultural information and general information. The result of the cultural probes method was a list of 36 user requirements, spread across the different clusters.

To rank-order the initial requirements by their relative importance and to obtain initial design ideas we carried out two focus group sessions [5]. Six research office workers from the Technical University of Eindhoven participated in the first and 10 in the second session. A general overview of the session was sent to each participant in advance. The participants in each group were asked to discuss the list of requirements that was provided and to select the requirements they found most important for stimulating social interaction at work. Thereafter, they were asked to propose a possible design for a system that would support social interaction among co-workers, using the selected requirements.

The focus groups helped us deduce a set of significant system requirements regarding the input, presentation and management of the content.

\section{The following are the selected user requirements:}

Personal information: Each group member should be able to share their personal history through the system or build a personal profile for other group members to view.

Organizational information: Company events, such as conferences, trips, excursions, and birthdays are important for stimulating social behavior that should be presented by the system. Other organizational information such as complaints, suggestions, and opinions can further engage the group members in interacting and socializing.

Ice breakers: Brain teasers, jokes, multiplayer games, open questions, traffic and weather reports all give opportunities for people to talk about, discuss and share.

\section{The following are the selected system requirements:}

System self-management: The system must be able to gather and manage some of content automatically. Content for sections like jokes can be gathered by the system from various sources on the web.

Attractive content presentation: The shown content must be appealing and inviting. All presented information must automatically change or cycle in appropriate periods to attract passersby. It must draw attention but not distract from a professional or social interaction that might be occurring.

Context awareness: The displayed content should be appropriate for the surrounding space. Group members can change or define the theme or appearance of the information for special events or occasions. Awareness of users would further help in providing most engaging content.

Extendibility: The system should allow users to change the content. Users should be able to adapt the system to suit particular group needs and preferences; for example, they should be able to define their own categories.

"Walk-up-and-use": System must be easy to use and self explanatory. First-time computer-savvy users should not need extra training to use the system effectively. 


\section{The System}

The main LID application, which shows the content on a touch screen, gathers content stored in the Basic Support for Collaborative Work (BSCW) database and from the web. The majority of the content is entered by group members through personal computers or other devices like Pocket PCs. The remaining content is retrieved automatically from the web by the LID application. The LID application makes frequent requests for user content, combines it with content from the web, and displays the interface on a large touch screen. Users registered in the workspace can access their workspace on the server and create, manage, and distribute documents, notes, links, etc [6]. The center of the LID screen displays the content selected. The tab bar section at the bottom of the screen contains ice breaker categories: jokes, games, questions, weather and traffic. The section on the right hand side of the screen contains the contents of the remaining user requirements, such as personal information sharing and organizational matter.

\section{Validation of User Requirements}

To validate user requirements a user evaluation was conducted. The goal was to find out if the user requirements were implemented in a way that is suitable for the working environment, and if the requirements would fit working routines and attract and keep the interest of the participants.

Set-up and procedure. The test took place at the Technical University of Eindhoven with 40 students of the post-graduate User System Interaction Program. The user test lasted for five days and was performed during working hours. To evaluate the use of the system, four different measures were taken. Interactions with the display were time-stamped and logged. Information regarding material uploaded to the BSCW system was logged, as well. Before and after the test participants filled in a questionnaire and 6 had a follow-up interview.

Results. Quantitative results: The total number of uploaded files was 46 . The highest number of uploads was in the category of pictures or images. The lowest number was 0 for the personal achievements category. Popular categories were also forum (6), non-university events (6), messages (5), and travel (5). The category music was added after a request by some of the participants and it recorded 31 uploaded music files.

Analysis of the logging data showed that most interactions took place on the first day of the evaluation (almost 350 interactions), after which the number of interactions decreased to a steady 150 per day. The most popular category was pictures (selected 111 times during the testing period) and the least popular category, Wikipedia (38 selections).

Interviews: Data regarding the participants' general impression was positive. All six interviewed participants found that the information presented on the screen was interesting, and presented in a suitable manner. They characterized the Large Interactive Display as funny, easy to use, and attractive. Automatic changes of the content were positively evaluated. The participants felt that the application did not change the group's social behavior, but it improved their communication. It also stimulated them 
to learn more about their colleagues and to share information about themselves. The participants described the application as a conversation starter, it made the atmosphere in the common room cozy and they would love to have it at their disposal in the future.

Questionnaire: A validated questionnaire for the evaluation of group communication was used to evaluate the effect of the system on the group interaction [7]. We found no significant changes in social interaction as perceived by the participants.

\section{Discussion and Conclusion}

The goal of this study was to design and evaluate an interactive system supporting social interaction at work. The added value of such a system for the work floor could be inferred from the analysis of the interaction logs and confirmed during interviews with the participants. However, the analysis of the study questionnaire showed no significant change regarding how participants felt about their social interactions before and after using the system, which may be explained that the evaluation period was too short to generate visible impact on social interactions in this particular group. Despite the limitations we were able to derive several interesting design implications for systems supporting social interaction at work. Ice breakers were a very important part of the system. They served a double function: not only did they attract people's attention to the system, but they also fostered interaction between users. We realize that each working group has different needs and requirements that are specific for this particular group and are not likely extendable to other groups. However, the categories defined in the present study could serve as a backbone for similar systems, although those systems should stay open for customization by their users.

\section{References}

1. Buunk, B.P., Verhoeven, K.: Companionship and Support at Work: a Microanalysis of the Stress-Reducing Features of Social Interaction. Basic and Applied Social Psychology 12, 243-258 (1991)

2. Bryant, S.: Workplace Friendships: Asset or Liability. Monster Work/Life Balance, http://content.monster.ca/9667_en-CA_p1.asp (viewed September 8, 2008)

3. Gaver, W., Dunne, T., Pacenti, E.: Cultural probes. Interact: New Visions Hum. Comput. Interact. 1, 21-29 (1999)

4. Hodson, R.: Work Life and Social Fulfillment: Does Social Affiliation at Work Reflect a Carrot or a Stick. Social Science Quarterly 81, 221-239 (2004)

5. Krueger, R.A., Casey, M.A.: Focus Groups: A Practical Guide for Applied Research. Sage Publications, Inc., Thousand Oaks (2000)

6. OrbiTeam Software Gmbh \& Co. BSCW - Version 4.4 Manual, OrbiTeam Sofware Gmbh \& Co. KG, Bonn, Germany

7. Widmeyer, W.N., Brawley, L.R., Carron, A.B.: The measurement of cohesion in sport teams: The group environment questionnaire. London: Sports Dynamics 1 (1985) 